\title{
MEMBRANE PERFORMANCE UNDER THE INFLUENCE OF MAGNETIC
} PRETREATMENT

I.J. LIN ${ }^{*}$ and S. NADIV ${ }^{* *}$

Mineral Engg. Dept. ${ }^{*}$ and Materials Engg. Dept.** Technion-Israel Institute of Technology

Haifa 32000, Israel

GENERAL

Membrane technology is undergoing expansion ${ }^{(1-3)}$ in both its design and application aspects. Extensive industrial R\&D activity is in progress with a view to indentification of selective membranes, to satisfactory long-term performance, and to cost reduction.

Membranes are widely used not only as means of separation and filtration, but also in chemical and physical processes- reactions, controlled release, extraction, heat transfer, energy conversion, energy cells, etc. A variety of types is known- organic and inorganic, made of polymers, biological tissues, glass, ceramics, or metals. Improved performance is achieved through diverse measures such as preliminary removal of suspensoids, application of reagents, periodic cleaning, and superimposition of force fields (ultrasonic, electric, mechanical vibration, etc.) on the membrane itself or in its vicinity. The last-named technique makes for improved heat- and mass-transfer, and also prevents clogging by deposits and permits flow-rate control and selectivity. in utilization of controlled magnetic fields in water treatment with a view to prevention of scale formation and removal of accumulated scale in industrial systems such as cooling towers, heat exchangers, air conditioners and solar installations- it was decided to test this technique as a means for minimizing deposi- 
tion of chemicals on membrane surfaces, with the attendant effect on their efficiency.

\section{EXPERIMENTAL}

A series of pilot-plant experiments was conducted on the reverseosmosis system shown in Fig. 1, under a long-term continuous regime.

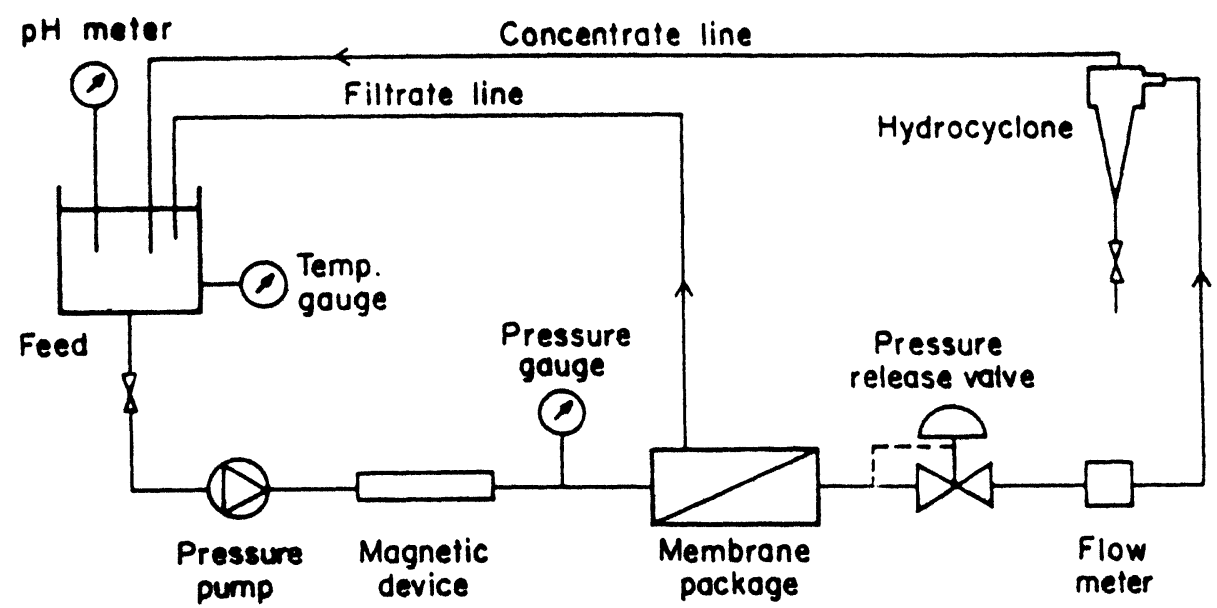

Figure 1- RO system for treatment of borate solution.

The system comprized a membrane separator (given package, $120 \mathrm{~cm}$ ), piping, a pressure- and a temperature-gauge and a flowmeter. Continuous removal of solid suspensoids, carried by the fluid or forming in it-was effected by means of a closed-bottom hydrocy- 
clone (Fig.2) ${ }^{(5)}$.

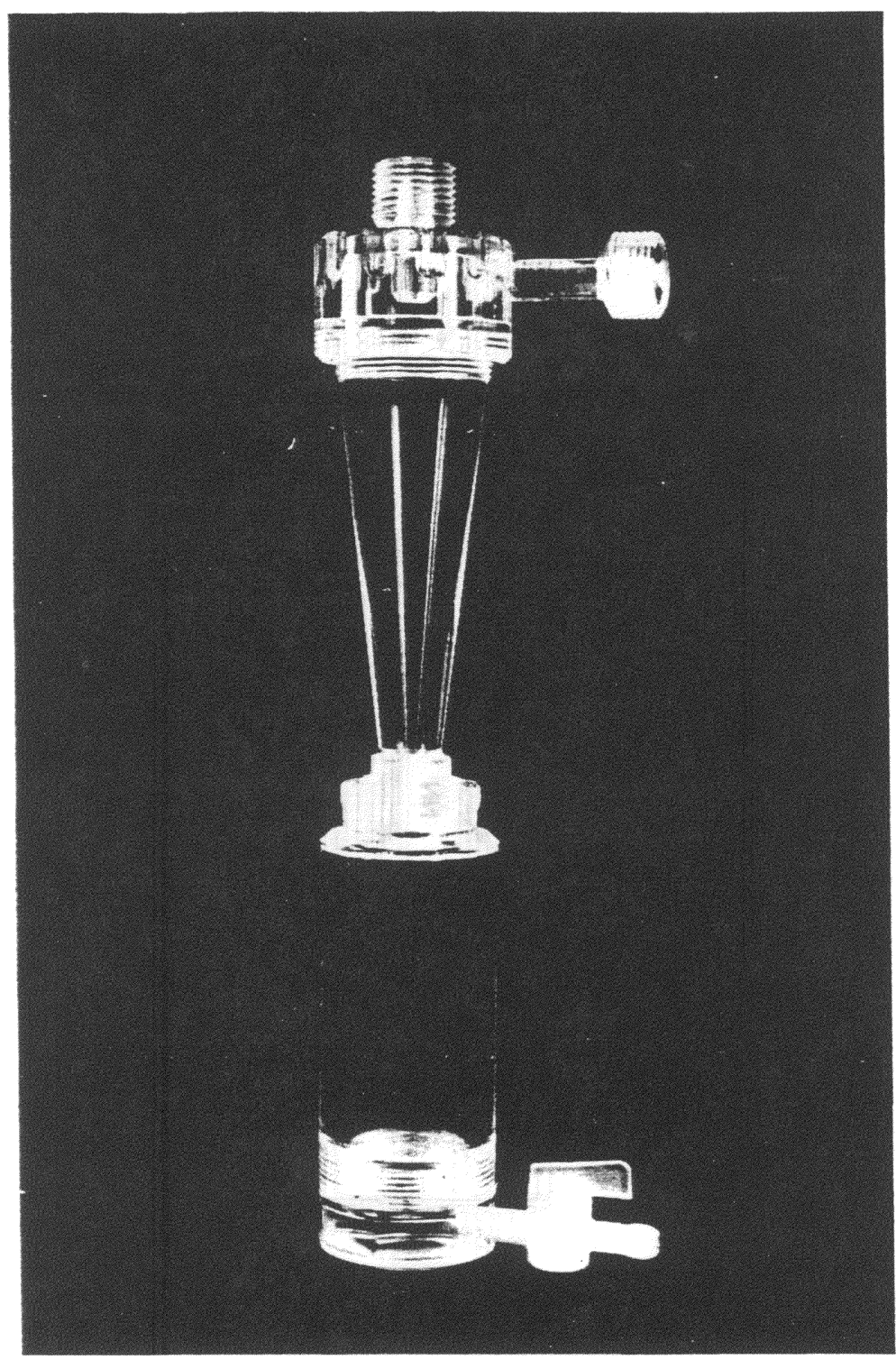

Figure 2- Closed-bottom hydrocylcone. 
A model RT 750k "superior" magnetic device (permanent magnet, four alternating poles), adapted for treatment of fluids with throughput up to $8 \mathrm{gal} / \mathrm{min}$, was installed as shown in Fig. 3 .

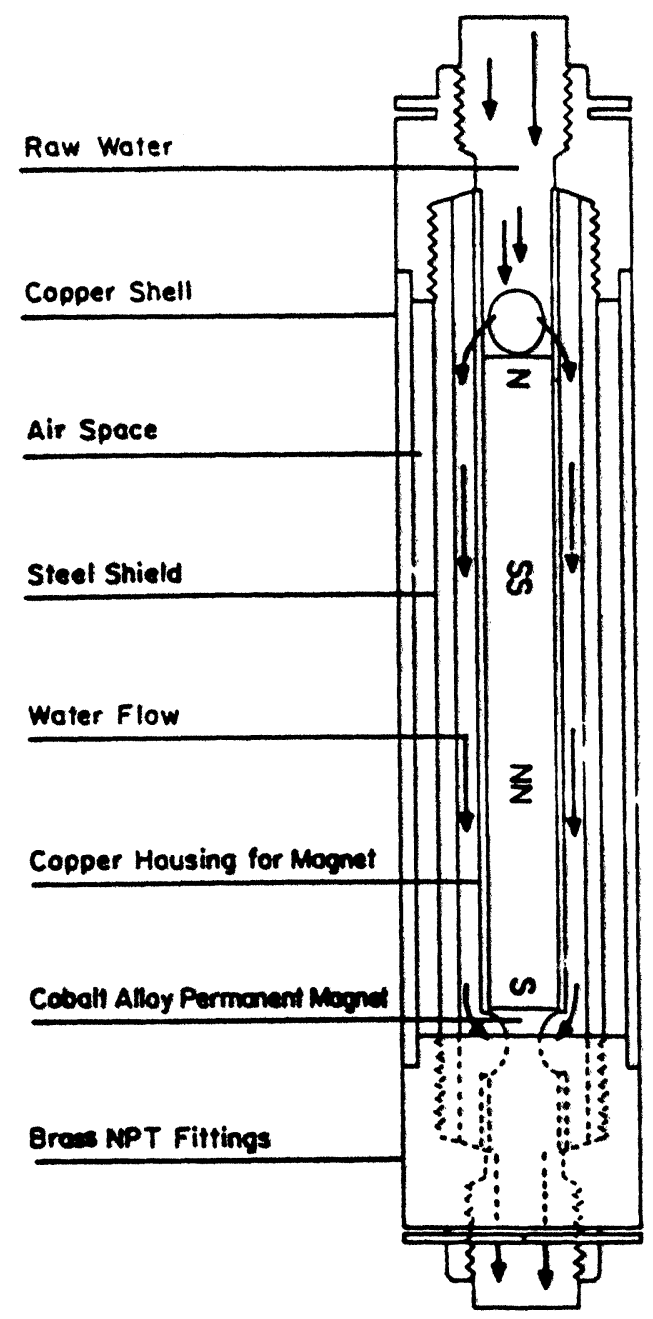

Figure 3- Magnetic device for water treatment. 
The system operates in a closed cycle, under variation of parameters such as type of concentration of the solutions, operation regime and type of equipment. The contribution of magnetic pretreatment can be evaluated through the following data:

(a) Solute element concentration on either side of the membrane.

(b) Electric conductivity of the fluid on either side of the membrane.

(c) Flow rate as function of time (permeability of the membrane).

(d) Degree of cleanliness of the membrane surface, and character and quantity of the deposit.

\section{FINDINGS}

The feed stock was calcium borate solution ( $\mathrm{pH}$ 10.5) in distilled and tap water. This alkaline environment favors deposition of the calcium in the tap water in the form of poorly-soluble salts (sulfate, carbonate) and as a boron complex. These solutions were used for determining the efficiency of magnetic pretreatment in reducing salt deposition on the membrane, and of the hydrocyclone in removing present and forming suspensoids in the flow path.

Results are summarized in Fig. 4, which presents the percentage of boron retention versus time, and indicates a definitely positive effect of the magnetic pretreatment over 750 hours of continuous operation in conjunction with the hydrocyclone.

Fig. 5 presents the flow rate versus time, and again a significant contribution of the magnetic pretreatment is indicated in the case of the tap-water solutions.

\section{CONCLUSION}

It was conclusively demonstrated that magnetic pretreatment inhibits and delays formation of clogging deposits on the membrane, and improves both the level of boron retention and the flow rate. Its mechanism is the same as in its application ${ }^{(6)}$ in preventing scale formation or in removing accumulated scale at solid-liquid inter- 
faces.

Solid suspensoids present in the feed stock or forming in the system are removed continuously and effectively by means of a closed-bottom hydrocyclone.

It was demonstrated that omission of the magnetic treatment in the present system results in drastic impairment of both the boron retention level and the flow rate-owing to deposition of poorly soluble calcium-containing crystals-with the attendant impairment of the performance.

Integration of combined magnetic treatment and $S-L$ separation in membrane techniques is recommended, specifically in the context of biological membranes.

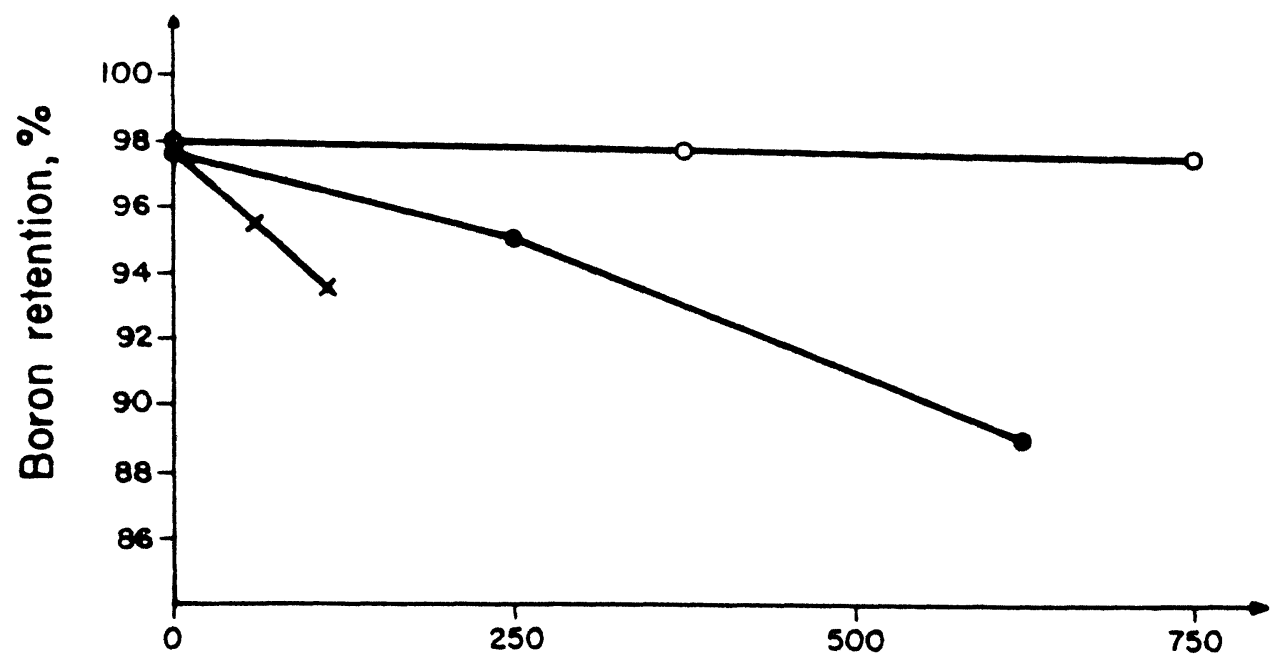

Time, hrs

Figure 4- Comparative behavior of MP-FO-10 membranes in $120 \mathrm{~cm}$ package.

0 without magnetic device (M.D.) and hydrocyclone (H.C.) distilated water

- with M.D. and H.C., tap water.

$x$ without M.D. and H.C., tap water. 


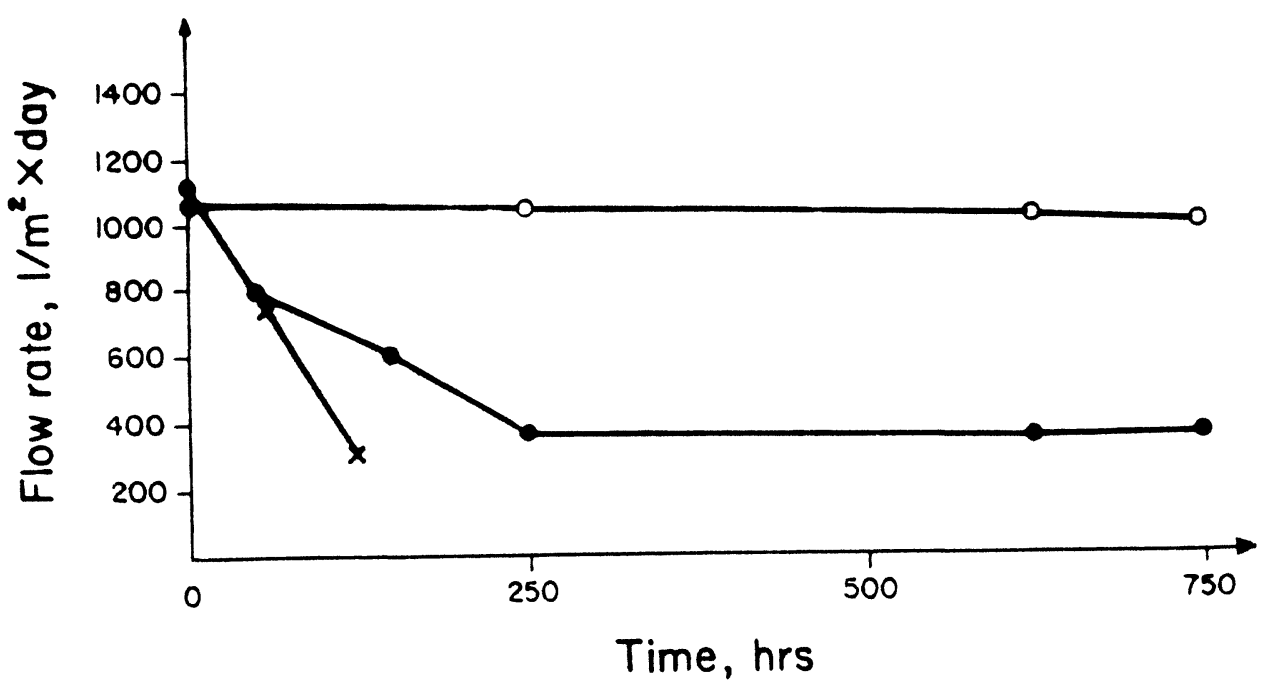

Figure 5- Filtrate flow rate vs. time-comparative pattern.

0 without magnetic device (M.D.) and hydrocylcone (H.C.), distilated water.

- with M.D. and H.C., tap water.

$x$ without M.D. and H.C., tap water.

\section{REFERENCES}

1. H.K. Lonsdale, The Growth of Membrane Technology, J. Mem Sci., $10,81,(1982)$.

2. A.J. Grodzinsky, Electric Field Control of Membrane Transport and Separations, Sep. and Purific. Methods, 14, No.1-40, (1985).

3. R.D. Noble, An Overview of Membrane Separation, Sep. Sci., and Tech., 22, Nos. $2 \& 3,731-743$, (1987).

4. I.J. Lin, S. Nadiv, The Influence of Low Intensity Magnetic Fields on Aqueous Solutions under Laminar Flow Regime, MERC Rep. 016-217, 57pp., April (1987).

5. I.J. Lin, Hydrocycloning-Thickening: Dewatering and Densification of Fine Particles, Sep. Sci., and Tech., 22, No. 4, 13271347,. (1987).

6. S. Srebrenik, S. Nadiv, I.J. Lin, Magnetic Treatment of WaterTheoretical Quantum Model, Colloid \& Surfaces-in press. 